\title{
Relativistic ejecta from XRF 060218 and the rate of cosmic explosions
}
A. M. Soderberg ${ }^{1}$, S. R. Kulkarni ${ }^{1}$, E. Nakar ${ }^{2}$, E. Berger ${ }^{3}$, P. B. Cameron ${ }^{1}$, D. B. Fox ${ }^{4}$, D. Frail ${ }^{5}$, A. Gal-Yam ${ }^{1}$, R. Sari ${ }^{1}$, S. B. Cenko ${ }^{6}$, M. Kasliwal ${ }^{1}$, R. A. Chevalier ${ }^{7}$, T. $\operatorname{Piran}^{8}$, P. A. Price ${ }^{9}$, B. P. Schmidt ${ }^{10}$, G. Pooley ${ }^{11}$, D.- S. Moon ${ }^{6}$, B. E. Penprase ${ }^{12}$, E. Ofek ${ }^{1}$, A. Rau ${ }^{1}$, N. Gehrels ${ }^{13}$, J. A. Nousek ${ }^{4}$, D. N. Burrows ${ }^{4}$, S. E. Persson ${ }^{3}$, and P. J. McCarthy ${ }^{3}$
${ }^{1}$ Caltech Optical Observatories 105-24, California Institute of Technology, Pasadena, CA 91125, USA
2 Theoretical Astrophysics 130-33, California Institute of Technology, Pasadena, CA 91125, USA
${ }^{3}$ Carnegie Observatories, 813 Santa Barbara St., Pasadena, CA 91101, USA
${ }^{4}$ Department of Astronomy, Pennsylvania State University, University Park, PA 16802, USA
${ }^{5}$ National Radio Astronomy Observatory, P.O. Box 0, Socorro, New Mexico 87801, USA
${ }^{6}$ Space Radiation Laboratory 220-47, California Institute of Technology, Pasadena, CA 91125, USA
${ }^{7}$ Department of Astronomy, University of Virginia, PO Box 3818, Charlottesville, VA 22903, USA
${ }^{8}$ Racah Institute of Physics, Hebrew University, Jerusalem 91904, Israel
${ }^{9}$ Institute for Astronomy, University of Hawaii, 2680 Woodlawn Drive, Honolulu, HI 96822, USA
10 RSAA, ANU, Mt. Stromlo Observatory, via Cotter Rd, Weston Creek, ACT 2611, Australia
${ }^{11}$ Mullard Radio Astronomy Observatory, Cavendish Laboratory, Cambridge CB3 0HE, UK
12 Pomona College Dept. of Physics \& Astronomy, 610 N. College Ave, Claremont, CA 91711, USA
${ }^{13}$ NASA Goddard Space Flight Center, Greenbelt, MD 20771, USA

Over the last decade, long-duration $\gamma$-ray bursts (GRBs) including the subclass of X-ray flashes (XRFs) have been revealed ${ }^{1-3}$ to be a rare variety of Type Ibc supernova (SN). While all these events result from the death of massive stars, the electromagnetic luminosities of GRBs and XRFs exceed those of ordinary Type Ibc SNe by many orders of magnitude. The essential physical process that causes a dying star to produce a GRB or XRF, and not just an SN, remains the crucial open question. Here we present radio and X-ray observations of XRF 060218 ( associated $^{4}$ with SN 2006aj), the second nearest $^{5,6}$ GRB identified to-date, which allow us to measure its total energy and place it in the larger context of cosmic explosions. We show that this event is 100 times less energetic but ten times more common than cosmological GRBs. Moreover, it is distinguished from ordinary Type Ibc SNe by the presence of $10^{48} \mathrm{erg}$ coupled to mildly-relativistic ejecta, along with a central engine (an accretion-fed, rapidly rotating compact source) which produces 
$\mathrm{X}$-rays for weeks after the explosion. This suggests that the production of relativistic ejecta is the key physical distinction between GRBs/XRFs and ordinary $\mathrm{SNe}$, while the nature of the central engine (black hole or magnetar) may distinguish typical bursts from low-luminosity, spherical events like XRF 060218.

On 2006 February 18.15 UT, the Burst Alert Telescope (BAT), a hard X-ray detector aboard the Swift satellite, detected ${ }^{5}$ an exceedingly long-duration ( $\Delta t \approx 2000 \mathrm{sec}$ ) transient. Within 153 seconds of the $\gamma$-ray trigger, the on-board X-ray Telescope (XRT) and Ultra-Violet Optical Telescope (UVOT) identified ${ }^{5}$ a counterpart coincident ${ }^{6}$ with a dwarf galaxy at $z=0.0335$. The XRT and BAT data show ${ }^{5}$ that the event peaked at a photon energy of $4.9 \mathrm{keV}$, thereby classifying this transient as an X-ray Flash, XRF 060218. Distinguished $^{7}$ by their soft X-ray dominated spectrum (peak energy, $E_{p} \lesssim 25 \mathrm{keV}$ versus $250 \mathrm{keV}$ ), the subclass of XRFs are otherwise similar (see ref. 8 and references therein) to GRBs in their observational properties.

Using the Very Large Array (VLA), we discovered a radio source at $\alpha(\mathrm{J} 2000)=03^{\mathrm{h}} 21^{\mathrm{m}} 39.68^{\mathrm{s}}$ and $\delta(\mathrm{J} 2000)=16^{\circ} 52^{\prime} 01.82^{\prime \prime}( \pm 0.02$ arcsec in each axis $)$, coincident with the UVOT position. Our monitoring of the radio source showed a power-law decay with $\alpha \approx-0.8$ through $t \approx 22 \mathrm{~d}$ (Table 1 ), similar to the decay of afterglows seen from GRBs; here $F_{\nu} \propto t^{\alpha}$ is the spectral flux density. Over the same period the XRT undertook intensive observations of the source in the X-ray band $(0.3-10 \mathrm{keV})$. We find the X-ray spectral flux density, $F_{\nu, X} \propto \nu^{\beta_{X}}$, is fit by $\beta_{X}=-2.2 \pm 0.2$ with an absorbing column density, $N_{\mathrm{H}}=3.9 \pm 0.4 \times 10^{21} \mathrm{~cm}^{-2}$, consistent with previously reported ${ }^{5,9}$ values.

Separately, we observed the source with the Advanced CCD Imaging Spectrometer (ACIS) instrument aboard the Chandra X-ray Observatory $(C X O)$. These observations began on 2006 February 26.78 and March 7.55 UT $(t \approx 8.8$ and 17.4 days) and lasted about 20 and $30 \mathrm{ks}$, respectively. The measured count rates are $(1.9 \pm 0.3) \times 10^{-3}$ and $(1.3 \pm 0.3) \times 10^{-3} \mathrm{~s}^{-1}$, respectively. Using the XRT model parameters stated above we derive $F_{X}=(4.5 \pm 1.4) \times 10^{-14}$ and $(2.8 \pm 0.9) \times 10^{-14} \mathrm{erg} \mathrm{cm}^{-2} \mathrm{~s}^{-1}$ for the unabsorbed flux values. The XRT-CXO data spanning the range from a few minutes to $17 \mathrm{~d}$ are well fit a simple power-law decay model with temporal index, $\alpha_{X}=-1.1$.

$\mathrm{XRF} 060218$ is most interesting because it is nearby, distance $d \approx 145 \mathrm{Mpc}$. Indeed it is second only to GRB $980425 / \mathrm{SN} 1998 \mathrm{bw}^{1}$ at just $36 \mathrm{Mpc}$. Similar to GRB 980425 , XRF 060218 is also associated ${ }^{4}$ with a Type Ic supernova explosion, SN 2006aj. The isotropic prompt energy release ${ }^{5} E_{\gamma, \text { iso }}=(6.2 \pm 0.3) \times 10^{49} \mathrm{erg}$, is at least 100 times fainter 
than typical GRBs but comparable to another nearby event, GRB 031203 ${ }^{10,11}(z=0.106)$. Similarly, the radio and X-ray luminosities are $10^{3}$ and $10^{2}$ times fainter than those of cosmological GRBs, respectively.

Radio observations directly probe the ejecta and environments of stellar explosions since the blastwave (velocity $v$ ) shocks the circumstellar medium and accelerates relativistic electrons which give rise to radio synchrotron emission. For radio sources dominated by synchrotron-self absorption, the brightness temperature is $T_{B} \lesssim 4 \times 10^{10} \mathrm{~K}$. As can be seen from Figure 1, at day 5 the radio emission peaks between $1.4 \mathrm{GHz}$ and $4.9 \mathrm{GHz}$. Applying the basic equipartition analysis (see ref. 2) we find, at this epoch, that the radius of the radio emitting region is $r \approx 3 \times 10^{16} \mathrm{~cm}$, the ejecta kinetic energy is $E_{K} \approx 2 \times 10^{48}$ erg and the circumburst particle density is $n \approx 5 \mathrm{~cm}^{-3}$. The blast wave thus expands with a Lorentz factor $\Gamma=\left(1-\beta^{2}\right)^{-1 / 2} \sim 2.3$; here $\beta \equiv v / c$.

The early, steady decay of the radio emission indicates ${ }^{12}$ that it cannot be attributed to a collimated jet directed away from our line-of-sight. Moreover, on a timescale, $t_{\mathrm{NR}} \approx 7.3\left(E_{\mathrm{K}, 48} / n_{0}\right)^{1 / 3}$ days, the blastwave becomes ${ }^{13}$ sub-relativistic $(\Gamma \beta<1)$ at which point it effectively assumes spherical geometry, even if the initial explosion was biconical. Independently, noting the absence of a "jet break" in the radio light-curve (to $22 \mathrm{~d}$ ) and applying the standard formulation ${ }^{14}$ we find the opening angle, $\theta_{j} \gtrsim 1.4$ radian. Thus, on several grounds, the radio data argue for a quasi-spherical ejecta with $10^{48} \mathrm{erg}$ coupled to mildly-relativistic material. In addition, our observations at 104 days show no evidence for a late-time increase in the radio flux, thus constraining the presence of additional ejecta components (off-axis jets; Figure 2) spreading into our line-of-sight.

As can be seen from Figure 1 the above synchrotron model is unable to explain the strong X-ray emission. Attributing the emission to scattering of SN optical photons by the mildly-relativistic ejecta requires an optical depth, $\tau \sim 10^{-4}$, too large to be produced by the shocked electrons which provide $\tau=n r \sigma_{T} \sim 10^{-7}$; here $\sigma_{T}$ is the Thomson cross section. We must therefore seek an entirely different origin for the observed X-rays.

At day 1, the steep X-ray spectrum roughly connects to the peculiar optical/UV component $\left(\beta_{O X} \sim-2\right)$ observed $^{5}$ to peak on this timescale. A similar steep nearIR spectrum was $\operatorname{seen}^{15}$ in GRB 031203 at $t=0.4 \mathrm{~d}$. Given that both GRB 031203 and XRF 060218 are $^{11,10}$ sub-energetic events, we suggest that this mysterious steep component is ubiquitous among sub-energetic GRBs and speculate that a central engine is the origin of this intense, long-lived emission. One particularly attractive possibility is a rapidly rotating (period, $P$ ) highly magnetized (field strength, $B$ ) neutron star, a magnetar. 
The spin down power, $\dot{E}=10^{45}(\mathrm{P} / 10 \mathrm{~ms})^{-4}\left(\mathrm{~B} / 10^{15} \mathrm{G}\right)^{2} \mathrm{erg} \mathrm{s}^{-1}$, can explain the peculiar optical to X-ray integrated luminosity at 1 day while the temporal evolution requires a braking index lower than three (magnetic dipole). We note that similarly low braking indices are measured for young Galactic pulsars (e.g. ref. 16 and references therein).

Combining the sky coverage and detection thresholds of $\gamma$-ray missions, we estimate the following sensitivity to the two exemplars of low energy events (GRB980425 and XRF 060218): $3.8 \times 10^{-3}\left(\right.$ BeppoSAX), $1.2 \times 10^{-3}\left(\right.$ HETE-2) and $3.7 \times 10^{-3} \mathrm{Gpc}^{3} \mathrm{yr}$ (Swift). Thus, the true rate of sub-energetic GRBs is $230_{-190}^{+490} \mathrm{Gpc}^{-3} \mathrm{yr}^{-1}$ (90\% confidence range; see Supplementary Information I), about 10 times more abundant than typical bright GRBs, ${ }^{17}$ for which we use a mean inverse beaming factor of $<f_{b}^{-1}>\sim 100$; here $f_{b} \equiv 1-\cos \theta_{j}$. Separately, we note that sub-energetic GRBs could not be strongly beamed or the true rate of such events would exceed the local $(d \lesssim 100 \mathrm{Mpc})$ rate $^{18,19}$ of Type Ibc supernovae, $9_{-5}^{+3} \times 10^{3} \mathrm{Gpc}^{-3} \mathrm{yr}^{-1}$.

Spectroscopy of the nearest GRB-associated supernovae (SNe 1998bw, 2003dh, 2003lw and now 2006aj) reveals (see ref. 4 and references therein) remarkably broad absorption lines (indicative of fast ejecta) and may suggest that all GRB-SNe are broad-lined (BL). Locally, BL events comprise ${ }^{20}$ five percent of SNe Ibc. Thus, the rate of BL events and sub-energetic GRBs are comparable, suggesting that all BL SNe harbor a long-lived central engine.

Radio observations of an extensive sample of 144 optically-selected local SNe Ibc, however, suggest ${ }^{21,12}$ a different picture (Figure 2). Not a single SN (BL or otherwise) shows strong early radio emission comparable to that seen in SNe 1998bw and 2006aj. Thus, we constrain the volumetric rate of such events to be $\lesssim 300 \mathrm{Gpc}^{-3} \mathrm{yr}^{-1}$ (see Supplementary Information II), consistent with the rate of sub-energetic GRBs inferred above. Focusing on the BL SNe, less than one in three are similar to GRBs, indicating that broad lines cannot be used as a reliable proxy for a central engine.

The commonality between the three nearest events $(980425,060218,031203)$ is their substantial $\left(E_{K} \gtrsim 10^{48} \mathrm{erg}\right)$ mildly-relativistic $(\Gamma \gtrsim 2)$ ejecta and a smooth pulse profile for the prompt emission. These two clues lead us to suggest that the primary physical distinction between GRBs/XRFs and ordinary supernovae is the velocity profile of the ejecta. For the latter, hydrodynamic collapse requires that the ejecta energy is concentrated at low velocities, $E_{K} \propto(\Gamma \beta)^{-5.2}$. In comparison, the shallow velocity profiles inferred for GRBs and XRFs indicate that some other agent (an engine) enables coupling of copious energy to to relativistic material (Figure 3). 
We conclude by noting that magnetars constitute 22 about $10 \%$ of the Galactic neutron star birth-rate, and thus a similar fraction of SNe Ibc. This rate is similar to that of the sub-energetic GRBs. Furthermore, magnetars produce long-lived emission (see ref. 23 and references therein) and have been suggested ${ }^{24}$ previously as candidate GRB progenitors. We therefore speculate that a magnetar central engine is what distinguishes sub-energetic GRBs from the cosmological bursts, which are thought to be powered by a black hole.

Received 11 June 2018; Accepted draft.

1. Galama, T. J., Vreeswijk, P. M., van Paradijs, J., Kouveliotou, C., Augusteijn, T. et al. An unusual supernova in the error box of the gamma-ray burst of 25 April 1998. Nature 395, 670-672 (1998).

2. Kulkarni, S. R., Frail, D. A., Wieringa, M. H., Ekers, R. D., Sadler, E. M. et al. Radio emission from the unusual supernova 1998bw and its association with the gamma-ray burst of 25 April 1998. Nature 395, 663-669 (1998).

3. Matheson, T., Garnavich, P. M., Stanek, K. Z., Bersier, D., Holland, S. T. et al. Photometry and Spectroscopy of GRB 030329 and Its Associated Supernova 2003dh: The First Two Months. Astrophys. J. 599, 394-407 December 2003.

4. Pian, E., Mazzali, P. A., Masetti, N., Ferrero, P., Klose, S. et al. Gamma-Ray Burst associated Supernovae: Outliers become Mainstream. Nature, submitted (astro-ph/0603530).

5. Campana, S., Mangano, V., Blustin, A. J., Brown, P., Burrows, D. N. et al. The shock break-out of GRB 060218/SN 2006aj. Nature, in press (astro-ph/0603279).

6. Mirabal, N., Halpern, J. P., An, D., Thorstensen, J. R. \& Terndrup, D. M. GRB 060218/SN 2006aj: A Gamma-Ray Burst and Prompt Supernova at $\mathrm{z}=0.0335$. Astrophys. J., submitted (astro-ph/0603686).

7. Heise, J., in’t Zand, J., Kippen, R. M. \& Woods, P. M. in Gamma-ray Bursts in the Afterglow Era (eds Costa, E., Frontera, F. \& Hjorth, J.) 16-+ 2001.

8. Soderberg, A. M., Kulkarni, S. R., Berger, E., Fox, D. B., Price, P. A. et al. A Redshift Determination for XRF 020903: First Spectroscopic Observations of an X-Ray Flash. Astrophys. J. 606, 994-999 May 2004.

9. De Luca, A. GRB 060218: analysis of the XMM-Newton observation. GRB Circular Network 4853, (2006).

10. Sazonov, S. Y., Lutovinov, A. A. \& Sunyaev, R. A. An apparently normal $\gamma$-ray burst with an unusually low luminosity. Nature 430, 646-648 August 2004. 
11. Soderberg, A. M., Kulkarni, S. R., Berger, E., Fox, D. W., Sako, M. et al. The sub-energetic $\gamma$-ray burst GRB 031203 as a cosmic analogue to the nearby GRB 980425. Nature 430, 648-650 August 2004.

12. Soderberg, A. M., Nakar, E., Berger, E. \& Kulkarni, S. R. Late-Time Radio Observations of 68 Type Ibc Supernovae: Strong Constraints on Off-Axis Gamma-Ray Bursts. Astrophys. J. 638, 930-937 February 2006.

13. Waxman, E. The Nature of GRB 980425 and the Search for Off-Axis Gamma-Ray Burst Signatures in Nearby Type Ib/c Supernova Emission. Astrophys. J. 602, 886-891 February 2004.

14. Sari, R., Piran, T. \& Halpern, J. P. Jets in Gamma-Ray Bursts. Astrophys. J. 519, L17-L20 July 1999.

15. Malesani, D., Tagliaferri, G., Chincarini, G., Covino, S., Della Valle, M. et al. SN 2003lw and GRB 031203: A Bright Supernova for a Faint Gamma-Ray Burst. Astrophys. J. 609, L5-L8 July 2004.

16. Lyne, A. G., Pritchard, R. S., Graham-Smith, F. \& Camilo, F. Very low braking index for the VELA pulsar. Nature 381, 497-498 (1996).

17. Schmidt, M. Luminosity Function of Gamma-Ray Bursts Derived without Benefit of Redshifts. Astrophys. J. 552, 36-41 May 2001.

18. Cappellaro, E., Evans, R. \& Turatto, M. A new determination of supernova rates and a comparison with indicators for galactic star formation. Astr. Astrophys. 351, 459-466 November 1999.

19. Dahlen, T., Strolger, L.-G., Riess, A. G., Mobasher, B., Chary, R.-R. et al. High-Redshift Supernova Rates. Astrophys. J. 613, 189-199 September 2004.

20. Podsiadlowski, P., Mazzali, P. A., Nomoto, K., Lazzati, D. \& Cappellaro, E. The Rates of Hypernovae and Gamma-Ray Bursts: Implications for Their Progenitors. Astrophys. J. 607, L17-L20 May 2004.

21. Berger, E., Kulkarni, S. R., Frail, D. A. \& Soderberg, A. M. A Radio Survey of Type Ib and Ic Supernovae: Searching for Engine-driven Supernovae. Astrophys. J. 599, 408-418 December 2003.

22. Gaensler, B. M., McClure-Griffiths, N. M., Oey, M. S., Haverkorn, M., Dickey, J. M. et al. A Stellar Wind Bubble Coincident with the Anomalous X-Ray Pulsar 1E 1048.1-5937: Are Magnetars Formed from Massive Progenitors? Astrophys. J. 620, L95-L98 February 2005. 
23. Hurley, K., Boggs, S. E., Smith, D. M., Duncan, R. C., Lin, R. et al. An exceptionally bright flare from SGR 1806-20 and the origins of short-duration $\gamma$-ray bursts. Nature 434, 1098-1103 April 2005.

24. Usov, V. V. Millisecond pulsars with extremely strong magnetic fields as a cosmological source of gamma-ray bursts. Nature 357, 472-474 June 1992.

25. Chevalier, R. A. Synchrotron Self-Absorption in Radio Supernovae. Astrophys. J. 499, 810-+ May 1998.

26. Granot, J. \& Sari, R. The Shape of Spectral Breaks in Gamma-Ray Burst Afterglows. Astrophys. J. 568, 820-829 April 2002.

27. Berger, E., Kulkarni, S. R., Pooley, G., Frail, D. A., McIntyre, V. et al. A common origin for cosmic explosions inferred from calorimetry of GRB030329. Nature 426, 154-157 November 2003.

28. Baron, E., Branch, D., Hauschildt, P. H., Filippenko, A. V. \& Kirshner, R. P. Spectral Models of the Type IC Supernova SN 1994I in M51. Astrophys. J. 527, 739-745 December 1999.

29. Mazzali, P. A., Deng, J., Nomoto, K., Pian, E., Tominaga, N. et al. A Neutron Star-driven XRF associated with SN 2006aj. Nature, submitted (astro-ph/0603567).

30. Tan, J. C., Matzner, C. D. \& McKee, C. F. Trans-Relativistic Blast Waves in Supernovae as Gamma-Ray Burst Progenitors. Astrophys. J. 551, 946-972 April 2001.

Correspondence should be addressed to A. M. Soderberg (e-mail:ams@astro.caltech.edu).

\section{Acknowledgements}

GRB research at Caltech is supported in part by funds from NSF and NASA. We are, as always, indebted to Scott Barthelmy and the GCN. The VLA is operated by the National Radio Astronomy Observatory, a facility of the National Science Foundation operated under cooperative agreement by Associated Universities, Inc. AMS and SBC are supported by NASA Graduate Research Fellowships. EB and AG acknowledge support by NASA through a Hubble Fellowship grant. DNB and JAN acknowledge support by NASA. 


\begin{tabular}{lcccccc}
\hline $\begin{array}{l}\text { Epoch } \\
(\mathrm{UT})\end{array}$ & $\begin{array}{c}\Delta t \\
(\text { days })\end{array}$ & $\begin{array}{c}F_{1.43} \\
(\mu \mathrm{Jy})\end{array}$ & $\begin{array}{c}F_{4.86} \\
(\mu \mathrm{Jy})\end{array}$ & $\begin{array}{c}F_{8.46} \\
(\mu \mathrm{Jy})\end{array}$ & $\begin{array}{c}F_{15.0} \\
(\mu \mathrm{Jy})\end{array}$ & $\begin{array}{c}F_{22.5} \\
(\mu \mathrm{Jy})\end{array}$ \\
\hline 2006 Feb 20.02 & 1.87 & - & $78 \pm 70$ & $453 \pm 77$ & - & - \\
2006 Feb 21.14 & 3.00 & - & - & $381 \pm 60$ & - & $250 \pm 52$ \\
2006 Feb 21.77 & 3.62 & - & - & - & $350 \pm 350$ & - \\
2006 Feb 21.97 & 3.83 & - & $287 \pm 56$ & $269 \pm 40$ & - & - \\
2006 Feb 22.99 & 4.85 & $25 \pm 25$ & $328 \pm 61$ & $280 \pm 47$ & - & - \\
2006 Feb 25.12 & 6.97 & $134 \pm 145$ & $80 \pm 47$ & $164 \pm 39$ & $46 \pm 141$ & - \\
2006 Feb 26.09 & 7.94 & - & $32 \pm 32$ & $30 \pm 30$ & - & - \\
2006 Feb 28.10 & 9.95 & - & - & $39 \pm 25$ & - & - \\
2006 Mar 2.23 & 12.08 & $70 \pm 70$ & - & - & - & - \\
2006 Mar 3.03 & 12.88 & - & - & $15 \pm 15$ & - & - \\
2006 Mar 6.89 & 16.74 & - & - & $75 \pm 13$ & - & - \\
2006 Mar 10.01 & 19.86 & - & - & $48 \pm 14$ & - & - \\
2006 Mar 12.11 & 21.96 & - & - & $87 \pm 39$ & - & - \\
2006 Mar 15.04 & 24.91 & - & - & $20 \pm 20$ & - & - \\
2006 Mar 20.86 & 30.71 & - & - & $32 \pm 20$ & - & - \\
2006 Mar 24.96 & 34.81 & - & - & $15 \pm 18$ & - & - \\
2006 Mar 26.85 & 36.70 & $69 \pm 69$ & $5 \pm 37$ & - & - & - \\
2006 Mar 31.89 & 41.74 & - & - & $22 \pm 22$ & - & - \\
2006 Apr 9.84 & 50.70 & - & - & $25 \pm 25$ & - & - \\
2006 Jun 2.67 & 104.52 & - & - & $17 \pm 21$ & - & -
\end{tabular}

\section{Table 1.}

Radio observations made with the Very Large Array (VLA) and the Ryle Telescope ${ }^{\dagger}$. We used the standard continuum mode with $2 \times 50 \mathrm{MHz}$ bands (VLA) and $350 \mathrm{MHz}$ bandwidth (Ryle). At 22.5 GHz we used referenced pointing scans to correct for the systematic 10-20 arcsec pointing errors of the VLA antennas. We used the extra-galactic sources 3C 48 (J0137+331) and 3C 147 (J0542+498) for flux calibration, while the phase was monitored using J0319+190 (VLA) and J0326+1521 (Ryle). The data were reduced and analyzed using the Astronomical Image Processing System. The flux density and uncertainty were measured from the resulting maps by fitting a Gaussian model to the afterglow emission. 
Figure 1: Radio and X-ray light-curves of XRF 060218. Radio measurements are summarized in Table 1. Upper limits are given as $3 \sigma$ (inverted triangles). Solid lines are models of synchrotron emission from a spherical shock expanding into a wind-blown circumstellar medium $\left(n \propto r^{-2}\right)$. At $t=5 \mathrm{~d}$ the radio spectrum peaks near $4 \mathrm{GHz}$ due to the synchrotron self-absorption frequency, $\nu_{a}$. We assume that the energy density is partitioned between the relativistic electrons (energy distribution $N(\gamma) \propto \gamma^{-p}$ with $p \approx 2.1$ ) and magnetic field as $\epsilon_{e}=\epsilon_{B}=0.1$. We find that $E_{K} \approx 2 \times 10^{48} \mathrm{erg}$ is coupled to ejecta with $\Gamma \approx 2.3$. The expansion, $r \propto t^{m}$, appropriate $^{25}$ for a core-collapse SN explosion with a distribution of ejecta velocities, is fit with $m \approx 0.85$. We infer a progenitor mass loss rate of $2 \times 10^{-7} \mathrm{M}_{\odot} \mathrm{yr}^{-1}$ (for wind velocity, $v_{w}=10^{3} \mathrm{~km} \mathrm{~s}^{-1}$ ). These parameters constrain the characteristic synchrotron frequency, $\nu_{m} \approx 0.3 \mathrm{GHz}$, and the synchrotron cooling frequency, $\nu_{c} \approx 10^{14} \mathrm{~Hz}$, at $t=5$ days and thus $\nu_{m}<\nu_{a}$; consistent with the observed radio spectrum (inset, solid grey curve). A nearly identical fit is obtained for a trans-relativistic GRB blastwave expanding into a constant density circumstellar medium ${ }^{26}$ for parameters: $E_{K} \approx 1.2 \times 10^{48} \mathrm{erg}, n=10^{2} \mathrm{~cm}^{-3}, \epsilon_{e}=\epsilon_{B}=0.1$ and $p=2.1$; in this case the mildly-relativistic ejecta is assumed to expand with a single bulk Lorentz factor. These values constrain the geometry of the ejecta to be effectively spherical, $\theta_{j} \gtrsim 1$. The X-ray flux (XRT=circles; $\mathrm{XMM}=$ encircled dot, scaled to XRT spectral model; $\mathrm{CXO}=$ squares) is significantly brighter than an extrapolation of the above model as evidenced by the unusually flat radio to X-ray spectral index, $\beta_{R X} \approx-0.5$ (inset, dashed line), and the steep X-ray spectrum $\beta_{X} \approx-2.2$ (inset, black line), instead of $\beta_{X} \sim-1.1$ for typical GRBs. We suggest that the integrated optical to X-ray luminosity $\left(10^{44} \mathrm{erg} \mathrm{s}^{-1} ; 2-10^{4} \mathrm{eV}\right)$ can be attributed to the spin down power of a magnetar. By day 5 , the optical/UV spectrum is dominated by the thermal SN emission (inset).

Figure 2: Radio observations for a large sample of local Type Ibc supernovae. Since 1999 we have been monitoring the radio emission from optically-selected $\mathrm{SNe}$ Ibc with the Very Large Array. We use radio luminosity as a proxy for mildly-relativistic ejecta to quantify the fraction of SNe Ibc powered by central engines. Our observations of $144 \mathrm{SNe}$ show that most $\mathrm{SNe}$ Ibc do not produce strong radio emission and therefore show no evidence for a central engine. For comparison, we include the radio afterglows for nearby $(z \lesssim 0.25)$ GRBs 980425 and 030329, and XRF 020903 all three of which show ${ }^{2,27,8}$ evidence for an engine-driven explosion. XRF 060218 is intermediate between GRBs and BL SN 2002ap, demonstrating that broad lines are not a reliable proxy for strong radio emission. Radio limits for other local broad-lined SNe (encircled triangles) show that less than one in three of these events may have a radio luminosity comparable to XRF 060218 or GRB 980425 (90\% confidence level). In addition, we show two 8.5 $\mathrm{GHz}$ model light-curves for a typical GRB viewed away from the collimation axis. Both models adopt typical GRB parameters (see ref. 12 and references therein) of $\Gamma=100, E_{K \text {,iso }}=10^{53} \mathrm{erg}$, $n=1 \mathrm{~cm}^{-3}, \epsilon_{e}=\epsilon_{B}=0.1$, and $p=2.1$. In the first model we assume that the observed $\gamma$-ray 
and radio emission are produced by a GRB jet viewed from an angle $\theta_{\text {obs }}=2 \theta_{j}$; here $\theta_{\text {obs }}$ is the angle between our line-of-sight and the jet axis. Under this framework, the observed prompt emission properties $\left(\Delta t, E_{p}, E_{\gamma, \text { iso }}\right)$ are related to the intrinsic values through the quantity $D \equiv\left[\Gamma\left(\theta_{\text {obs }}-\theta_{j}\right)\right]^{-2}$. For $D \sim 0.02$, the intrinsic properties for XRF 060218 would be typical for GRBs: $\Delta t \sim 40 \mathrm{sec}, E_{p} \sim 250 \mathrm{keV}, E_{\gamma \text {,iso }} \sim 10^{53} \mathrm{erg}$, and $\theta_{j} \sim 4^{\circ}$. The resulting off-axis model (dotted line) is a factor of $10^{3}$ brighter than the observed XRF 060218 radio light-curve and can therefore be ruled out. In the second model, we assume that in addition to the quasi-spherical mildly-relativistic ejecta component producing the observed radio emission, XRF 060218 also harbors a strongly collimated relativistic jet directed significantly away from our line-of-sight. In this scenario, we expect ${ }^{13,12}$ a late-time radio re-brightening as the jet becomes non-relativistic and spreads sideways into our line-of-sight. Adopting $\theta_{j}=4^{\circ}$ we find that our latest radio limit (104 days; black triangle) rules out an off-axis GRB with $\theta_{\text {obs }} \lesssim 60^{\circ}$ (dash-dot line). We conclude that the XRF 060218 ejecta was quasi-spherical and intrinsically sub-energetic.

Figure 3: Energy as a function of velocity for GRBs, XRFs, and SNe Ibc. Optical data (small dark circles) probe (see refs. 28,29 and references therein) the slowest ejecta in supernova explosions which typically carry the bulk of the kinetic energy $\left(E_{K}=0.3 M_{\mathrm{ej}} v_{\mathrm{ej}}^{2} \sim 10^{51} \mathrm{erg}\right.$ ). On the other hand, radio observations (large light circles) trace $27,2,11,21,25$ only the fastest ejecta in the explosion. For GRBs 030329 and $031203, \Gamma \propto t^{-3 / 8}$; we adopt the bulk velocity of the relativistic ejecta at day 1 as inferred from radio modeling. For GRB 980425, XRF 060218, SN 20020ap and SN 1994I the bulk velocity is roughly constant on the timescale probed by the radio observations; we adopt the velocity at the radio peak time. Standard hydrodynamic collapse results ${ }^{30}$ in a kinetic energy profile, $E_{K} \propto(\Gamma \beta)^{-5.2}$ (grey line), and thus a negligible fraction of the kinetic energy may be coupled to mildly-relativistic ejecta, consistent with the radio observations of local Ibc SNe 1994I and 2002ap. In the case of typical GRBs (e.g. GRB 030329), however, the kinetic energy of the mildly-relativistic ejecta is nearly comparable to that of the slower material indicating the presence of a central engine. Since the origin of the relativistic flow is separate from the SN, there is probably not a continuous distribution of matter between the two data points but rather distinct ejecta components. Sub-energetic bursts such as XRF 060218 are intermediate between these two classes and may indicate that their central engines are different than those of typical GRBs. We conclude that the minimum criteria for producing GRBs and XRFs is a mildly-relativistic $(\Gamma \gtrsim 2)$, quasi-spherical ejecta carrying at least $10^{48} \mathrm{erg}$. 


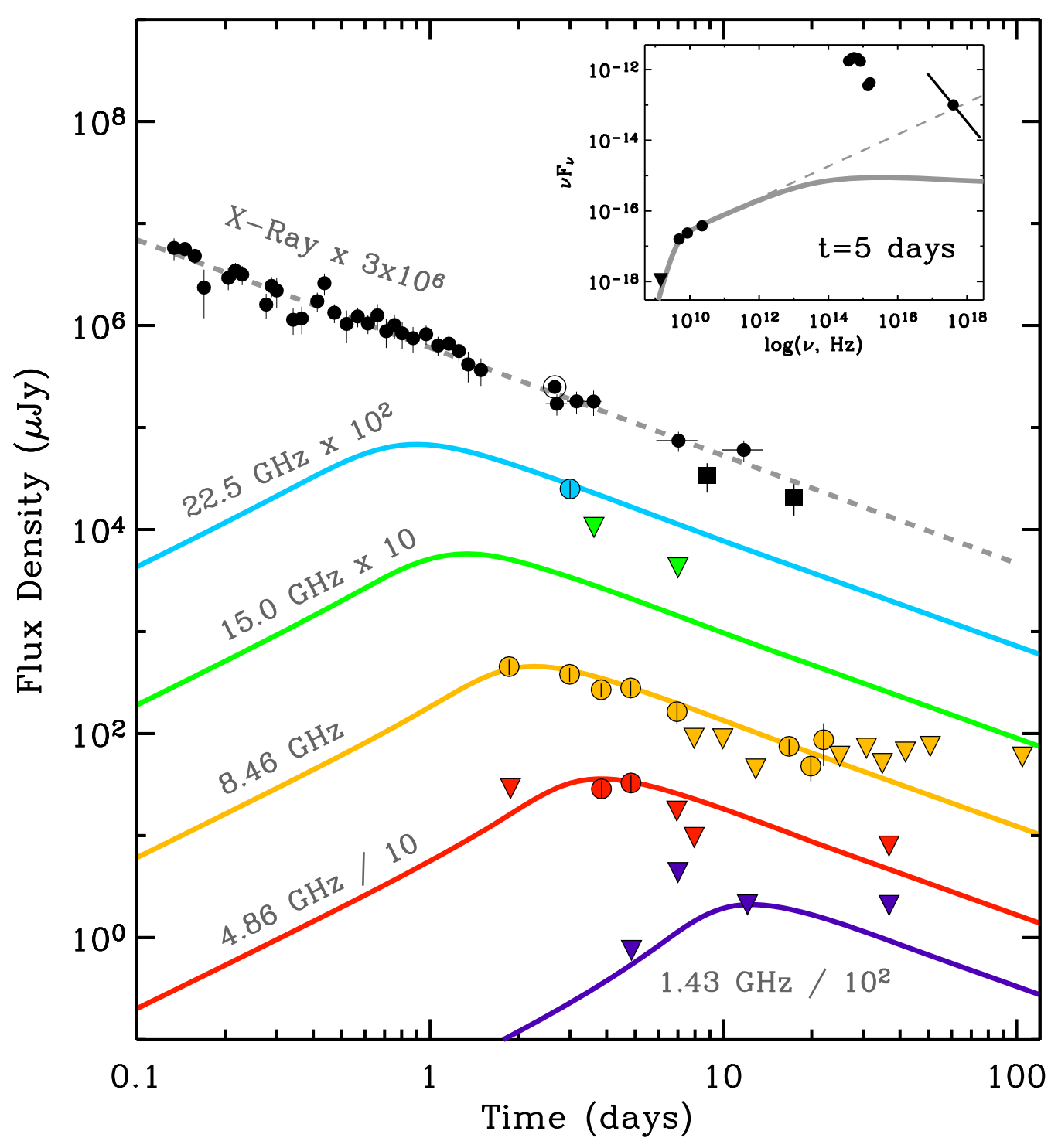

Figure 1. 


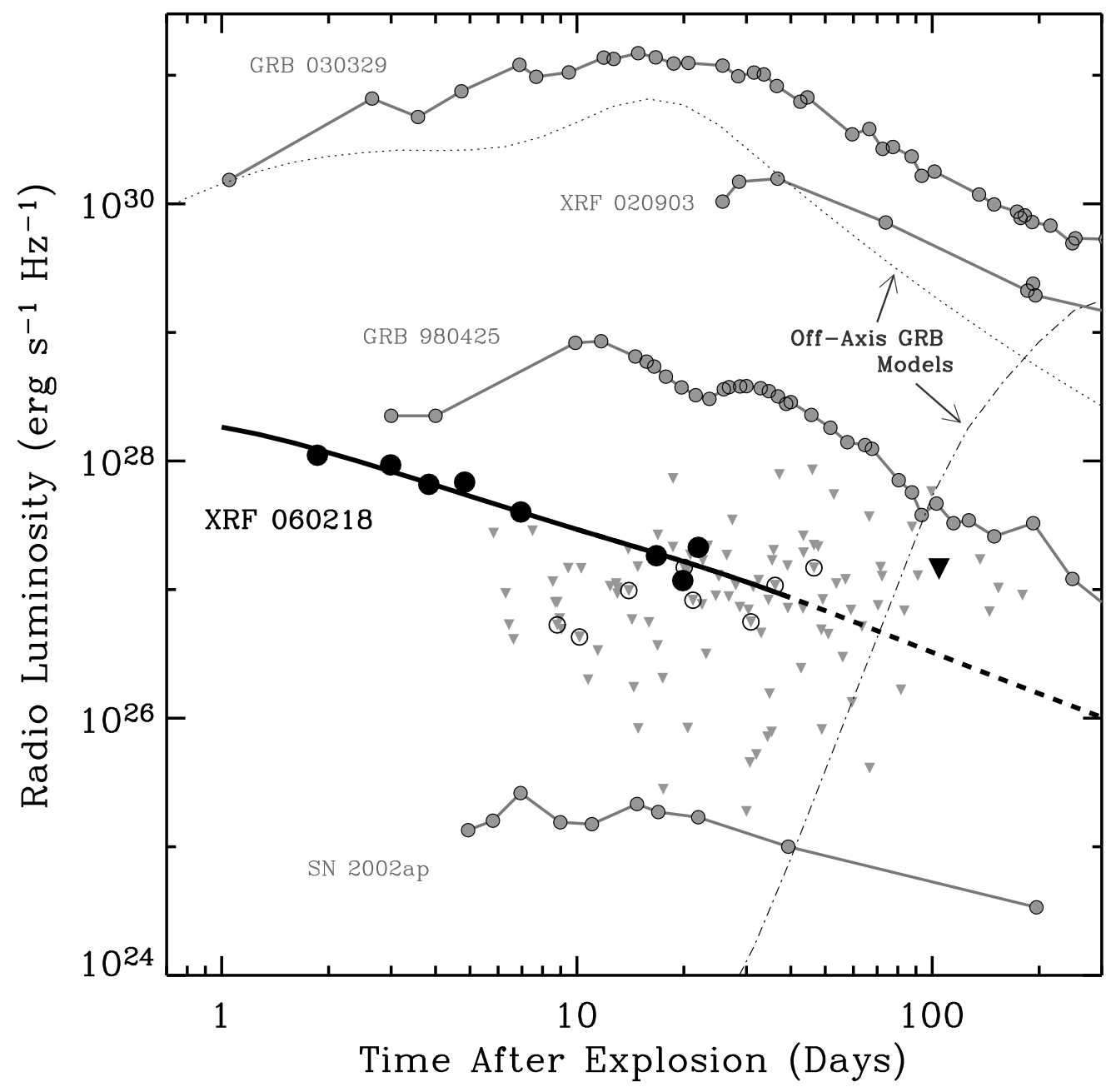

Figure 2. 


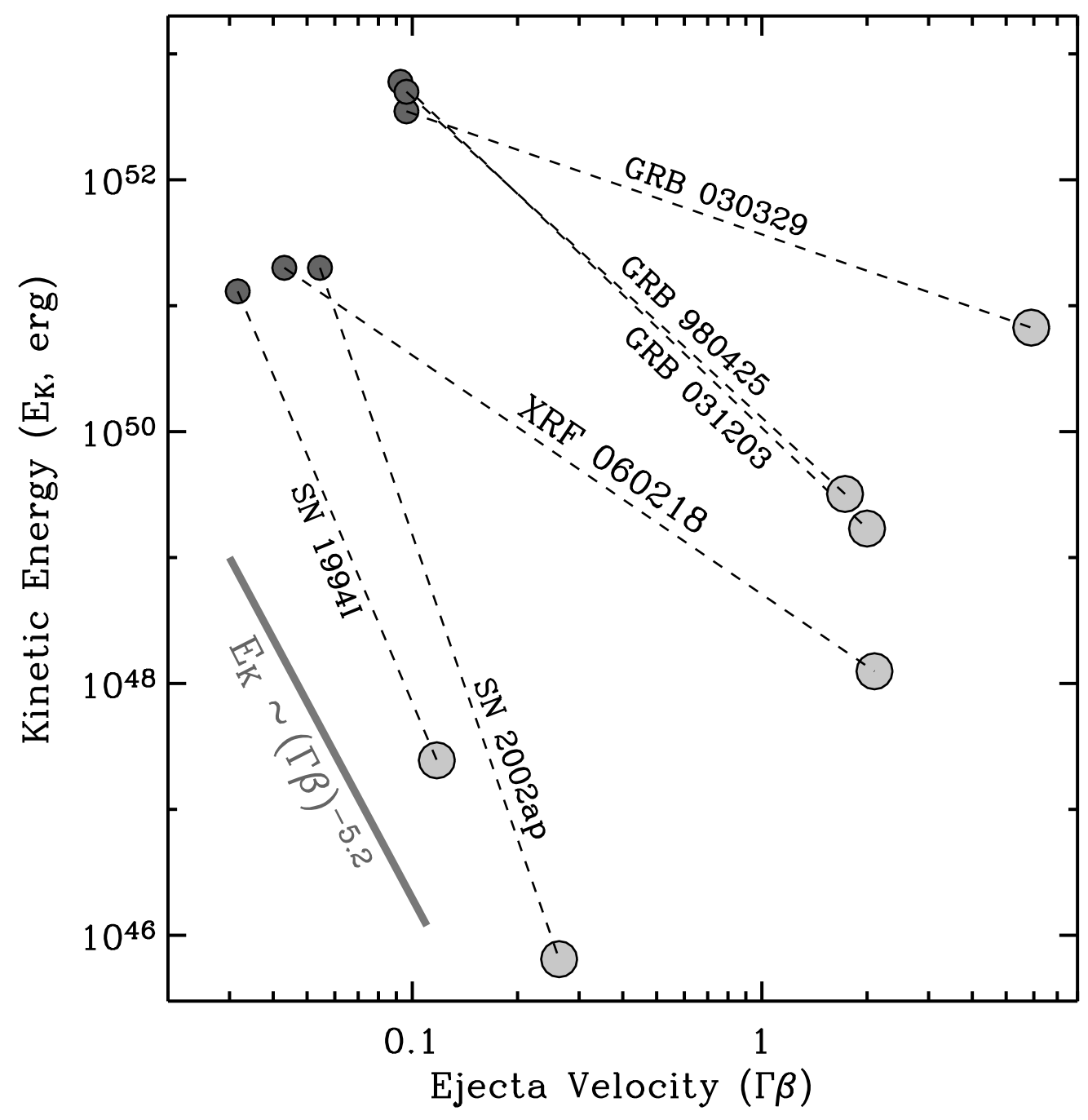

Figure 3. 


\section{SUPPLEMENTARY INFORMATION}

I. Estimates for the Rate of Sub-energetic GRBs: To estimate the rate of sub-energetic events similar to GRB 980425 ( $d=36.1 \mathrm{Mpc})$ and XRF 060218 ( $d=145 \mathrm{Mpc}$ ), we consider only the satellite instruments with precise localization capability: BeppoSAX Wide Field Cameras (WFC), High Energy Transient Explorer 2 (HETE-2) Wide Field X-ray Monitor (WXM), and Swift Burst Alert Telescope (BAT). Inclusion of INTEGRAL would not significantly affect our results due to its low GRB detection rate. For these three instruments we adopt the detection threshold curves calculated by Band (2003, ApJ, 588, 945; 2006, ApJ, 644, 378) in units of peak photon flux per second $\left(F_{\text {peak }} ; 1-1000 \mathrm{keV}\right)$ as a function of the $\nu F_{\nu}$ spectral peak energy, $E_{\text {peak }}$. We assume the shape of the prompt emission spectrum is fit by a broken power-law (Band et al. 1993, ApJ, 413, 281) such that $F_{\nu} \propto \nu^{\alpha}\left(F_{\nu} \propto \nu^{\beta}\right)$ for $h \nu<E_{\text {peak }}\left(h \nu>E_{\text {peak }}\right)$. Using the observed spectral parameters $\left(F_{\text {peak }}, E_{\text {peak }}, \alpha, \beta\right)$ we calculate the sensitivity of each instrument to each of the two events.

For GRB 980425, $F_{\text {peak }} \approx 3 \times 10^{-7} \mathrm{erg} \mathrm{cm}^{-2} \mathrm{~s}^{-1}(24-1820 \mathrm{keV}$; Galama et al. 1998, Nature, $395,670) E_{\text {peak }} \approx 160 \mathrm{keV}, \alpha \approx-0.27$ and $\beta \approx-2$ (Jimenez, Band \& Piran 2001, ApJ, 561, 171). Extrapolating to the $1-1000 \mathrm{keV}$ band, we find that the peak photon flux is $F_{p} \approx 8.4 \mathrm{ph} \mathrm{cm}^{-2} \mathrm{~s}^{-1}$ and thus the event could be detected to 120 (WFC), 60 (WXM) and 130 (BAT) Mpc.

For XRF 060218, $F_{\text {peak }} \approx 5 \times 10^{-8} \mathrm{erg} \mathrm{cm}^{-2} \mathrm{~s}^{-1}(15-150 \mathrm{keV}$; Campana et al. 2006, Nature, submitted) $E_{\text {peak }} \approx 4.9 \mathrm{keV}, \beta \approx-1.5$ and $\alpha$ is not constrained (15-150 keV; Campana et al. 2006, Nature, submitted). We estimate $F_{p} \approx 1.9 \mathrm{ph} \mathrm{cm}^{-2} \mathrm{~s}^{-1}$ and therefore XRF 060218 could be detected to 220 (WFC), 110 (WXM) and 180 (BAT) Mpc. We note that unlike other missions, the BAT detection threshold is significantly lower (factor of $\sim 3$ ) for unusually long duration events such as XRF 060218.

Next we estimate the effective monitoring time, $T_{m}$ of each of the missions assuming their sky coverage, $S$ and operation time, $T$. For the two Wide Field Cameras $S=2 \times 0.123=0.246 \mathrm{sr}$ (Band, 2003, ApJ, 588, 945) and $T=4 \mathrm{yrs,} \mathrm{and} \mathrm{for} \mathrm{WXM} S=0.806$ sr and $T=3 \mathrm{yrs}$ (Guetta et al., 2004, ApJ, 615, L73). For BAT, $S=2$ sr and $T=1$ yr (S. Barthelmy, private communication). Thus we find monitoring times, $T_{m}=(T / 4 \pi) S$, of 0.08 (WFC), 0.19 (WXM), and 0.16 (BAT) yrs.

We estimate the sensitivity of these instruments to each of the events as $T_{m} \times V$; here $V$ is the volume to which each event could be detected. Adopting the larger of the two sensitivities for each instrument we find $3.8 \times 10^{-3}$ (WFC), $1.2 \times 10^{-3}(\mathrm{WXM})$ and $3.7 \times 10^{-3}(\mathrm{BAT}) \mathrm{Gpc}^{3} \mathrm{yr}$. Summing the sensitivities, we find that the rate of sub-energetic events is $230_{-190}^{+490} \mathrm{Gpc}^{-3} \mathrm{yr}^{-1}$ where the errors are dominated by the $90 \%$ Poisson statistics for two detections (Gehrels, 1986, ApJ, 303, 336).

\section{Estimates for the rate of Type Ibc supernovae like GRB 980425 and XRF 060218 :}

To estimate the rate of SNe Ibc with strong, early radio emission comparable to that observed for 
sub-energetic bursts we only consider the 75 events (out of 144 optically-selected local SNe Ibc) with $3 \sigma$ upper limits fainter than the observed GRB 980425 and XRF 060218 light-curves at that same epoch. We then assume various values for the true fraction of SNe Ibc with radio emission comparable (or higher) to that of XRF 060218 and GRB 980425 and determine the probability of finding null-detections for all 75 events for each assumed fraction. Larger fractions are ruled out with higher confidence. At $90 \%$ confidence, we rule out the scenario where $\gtrsim 3 \%$ of $\mathrm{SNe}$ Ibc are as radio bright as XRF 060218 and GRB 980425. Adopting the local rate of SNe Ibc, $9_{-5}^{+3} \times 10^{-3} \mathrm{Gpc}^{-3} \mathrm{yr}^{-1}$, as measured by Cappellaro et al. (1999, Astr. Astrophysics, 351, 459) and Dahlen et al. (2004, ApJ, 613, 189), we conclude that the volumetric rate of events like GRB 980425 and XRF 060218 is less than $3 \%$ of the local SNe Ibc sample, or $\lesssim 300 \mathrm{Gpc}^{-3} \mathrm{yr}^{-1}$.

Repeating this analysis for the subset of broad-lined SNe Ibc, we find that at $90 \%$ confidence we can rule out the scenario where $\gtrsim 30 \%$ of local, optically selected BL SNe Ibc produce radio emission similar to that observed for GRB 980425 and XRF 060218. 